\section{The role of the strain elastosonography in the evaluation of canine hypoechoic splenic lesions}

\section{Gabriele Barella', Matteo Lodi², Stefano Faverzani'}

Milan State University, Dept. of Health, Animal Science and Food Safety, Milan, Italy

Practitioner, Milan, Italy

In human medicine elastosonography is helpful in differentiating benign from malignant lesions in several organs.The elastosonography has been described in veterinary medicine. Some authors found that elastosonography was not helpful in differentiating canine splenic nodules. This study aims to assess the repeatability and reproducibility of elastosonography in the evaluation of splenic nodules and to verify if it can differentiate benign from malignant splenic lesions in dogs.

Twenty-two dogs presenting a single splenic hypoechoic lesion less than $4 \mathrm{~cm}$ width, underwent ultrasonographic evaluation (two-dimensional ultrasound and strain elastosonography with a 7,5-13 MHz linear transducer). The Strain Ratio (SR or rather $z 2 / z 1$; where $z 1$ represented the entire lesion and $z 2$ a same size portion of normal splenic parenchyma at the same depth) and Hardness Value (HV; percentage of the lesion having $>50 \%$ of tissue classified as hard) were calculated for all the lesions. The repeatability and the reproducibility of this method were assessed calculating the Coefficient of Variability (CV) and the $\mathrm{K}$ of Cohen respectively. The analysis of the variance (ANOVA) and the Fisher exact test were used to verify the differences between the SR values and HV of benign versus malignant lesions. The cut off values for the Fisher test were arbitrarily set at 1,5 for the SR and at $70 \%$ for $\mathrm{HV}$; the Sensibility (Se) and Specificity (Sp) were also calculated. The Odds ratio was also calculated considering malignancy as bad outcome; lesions having a SR > 1,5 and lesions having an $\mathrm{HV}>$ $70 \%$ were considered in the exposed group.

Splenic nodules were diagnosed with cytology or histopathology as: extramedullary hematopoiesis (2), nodular iperplasia (13), hemangiosarcoma (5) and round cell neoplasia (2). The SR and HV of benign lesions were statistically different from those of malignant lesions $(p<0,05)$. Malignant lesions tended to have a $S R>1,5$ ( $p=0,001$; Se $71 \%$, Sp 99,9\%) and an $\mathrm{HV}>70 \%(p=0,005$; Se $100 \%$, Sp 67\%). The correlation between $S R>1,5$ and malignancy was statistically significant (OR, 63,8; 95\% Cl, 2,6238-1551,3391, $p=0,01)$ as the one between HV $>70 \%$ and malignancy (OR, 28,6; CI 95\% 1,3657$600,4393, p=0,03)$. This technique was repeatable and reproducible (CV 0,08 $\pm 0,03$ and $K=1$ for the $S R$; CV 0,08 $\pm 0,05$ and $\mathrm{K}=0,66$ for the $\mathrm{HV}$ ).

Elastosonography can differentiate malignant from benign canine hypoechoic splenic lesions less than $4 \mathrm{~cm}$ width.

\section{Radiographic femoral varus measurement is affected unpredictably by femoral rotation}

\section{James Miles}

Faculty of Health and Medical Sciences, University of Copenhagen, Frederiksberg, Denmark

Radiographic measurements of femoral varus are frequently used to determine if significant femoral deformity is present, and to calculate the required correction. The varus angle is defined as the angle between the proximal femoral long axis (PFLA) and the perpendicular to a line tangential to the distal femoral condyles. Ideal positioning of the femur has been defined as zero elevation relative to the radiographic plane and zero rotation along the long axis of the femur, but this may be difficult to obtain in practice. The clinician may thus be tempted to determine varus values from less than ideal radiographs.

Fourteen right femora were positioned at zero elevation (supracondylar eminence at same distance from the radiographic plate as the lesser trochanter, as defined in previous studies). The caudal aspects of the femoral condyles were placed in contact with the radiography cassette $\left(0^{\circ}\right.$ rotation), and subsequently rotated internally and externally by $5^{\circ}$ and $10^{\circ}$ using plastic wedges. Accuracy of rotation was within $+1^{\circ}$. Digital radiographs were obtained at each position. Varus angles were measured using ImageJ, employing two definitions of PFLA.

Mean varus angles increased with $10^{\circ}$ of either internal or external rotation with both PFLA definitions, but at $5^{\circ}$ increased slightly with one definition and decreased with the other. Scatter plots indicated that not all femora exhibited the same trend in change in varus angle. Significant differences $(p<0.05)$ were observed between varus measurements for the different PFLA definitions and between rotation angles.

The effect of rotation on varus angle measurements in these femoral specimens contradicts a previous report using CT. The most probable explanation is the difference in femoral positioning: the CT study used a slightly elevated position compared to that in this study, resulting in better visualisation of the condylar articular surfaces. Zero elevation frequently results in the articular surfaces being obscured by the trochlear ridges, even when ideally positioned.

In conclusion, femoral varus measurements vary unpredictably as a result of femoral rotation and this effect is likely further influenced by femoral elevation. 